TM-2152

\title{
BETATRON TUNE SHIFTS AND LASLETT IMAGE COEFFICIENTS
}

\author{
K.Y. Ng \\ Fermi National Accelerator Laboratory, ${ }^{*}$ \\ P.O. Box 500, Batavia, IL 60510
}

(May 2001)

\begin{abstract}
The complicated expressions of betatron tune shifts in terms of Laslett image coefficients are explained. The expressions of image coefficients for a centered or off-centered beam inside an elliptical or rectangular vacuum chamber are gathered. Typos in earlier literature are corrected.
\end{abstract}

${ }^{*}$ Operated by the Universities Research Association, Inc., under contract with the U.S. Department of Energy. 


\section{INTRODUCTION}

The incoherent and coherent betatron tune shifts of a beam inside the vacuum chamber of an accelerator ring can be expressed in term of Laslett image coefficients [1]. Unfortunately, these expressions are not easy to understand. The Laslett image coefficients for an off-centered line beam inside a beam pipe with elliptical or rectangular cross section have been studied in detail $[2,3,6]$. Unfortunately, elliptical functions are involved and these expressions are complicated. Even a single typo can often render an expression completely useless. In this note, we are going to gather first the expressions for tune shifts and try to explain each term in as much detail as possible. Next, we gather all the useful expressions for the image coefficients together with the method of evaluation. Most of the time, only the special case when the beam is at the center of the beam pipe is of interest and the image coefficients can be simplified tremendously. The special cases of a vacuum chamber with a circular cross section or consisting of two infinite horizontal plates are also included. In addition, most typos will be corrected.

\section{EXPRESSIONS FOR TUNE SHIFTS}

For a charged particle beam, the vertical and horizontal, incoherent and coherent betatron tune shifts are

$$
\begin{array}{ll}
\Delta \nu_{\text {incoh }}^{V, H}=-\frac{N r_{0} R}{\pi \gamma \beta^{2} \nu_{0}^{V, H}}\left[\left(\frac{1-\chi_{e}-\beta^{2}}{B}+\beta^{2}\right) \frac{\epsilon_{1}^{V, H}}{h^{2}}+\mathcal{F} \beta^{2} \frac{\epsilon_{2}^{V, H}}{h_{m}^{2}}+\left(1-\chi_{e}-\beta^{2}\right) \frac{\epsilon_{\mathrm{spch}}^{V, H}}{a_{V}^{2}}\right], \\
\Delta \nu_{\text {coh }}^{V, H}=-\frac{N r_{0} R}{\pi \gamma \beta^{2} \nu_{0}^{V, H}}\left[\left(\frac{1-\chi_{e}-\beta^{2}}{B}+\beta^{2}\right) \frac{\xi_{1}^{V, H}}{h^{2}}+\mathcal{F} \beta^{2} \frac{\xi_{2}^{V, H}}{h_{m}^{2}}\right] \quad \text { penetrating }, \\
\Delta \nu_{\text {coh }}^{V, H}=-\frac{N r_{0} R}{\pi \gamma \beta^{2} \nu_{0}^{V, H}}\left[\frac{1-\chi_{e}-\beta^{2}}{B} \frac{\xi_{1}^{V, H}}{h^{2}}+\beta^{2} \frac{\epsilon_{1}^{V, H}}{h^{2}}+\mathcal{F} \beta^{2} \frac{\epsilon_{2}^{V, H}}{h_{m}^{2}}\right] \quad \text { non-penetrating },
\end{array}
$$

where penetrating and non-penetrating imply whether the ac magnetic field coming from the betatron motion of the beam can penetrate or cannot penetrate the wall of the vacuum chamber. We pick out betatron motion, because the dc magnetic field from the stationary beam will always penetrate the vacuum chamber while the ac magnetic field from the high-frequency beam bunching will always be non-penetrating. If the betatron tune is near a half integer, the ac magnetic field will have frequency approximately 
half the revolution frequency ${ }^{\dagger}$ and will mostly be not able to penetrate the vacuum chamber. Thus, Eq. (2.3) is also called the half-integer formula. However, when the betatron tune is near an integer, the magnetic field will have low frequency and will mostly penetrate the vacuum chamber. Therefore, Eq. (2.2) is also called the integer formula. Of course, whether the ac magnetic field can penetrate the walls of the vacuum chamber or not depends also on the thickness of the walls. The integer formula is therefore applied to vacuum chamber of very thin walls while the half-integer formula is applied to vacuum chamber of very thick wall. Such complication does not arise in the incoherent tune shifts, where the restoring force comes from the particle beam stationary in the transverse directions. Thus, the magnetic field from the beam is dc and is always penetrating. For those readers who are interested in the details, each term of the above expressions is explained carefully in the Appendix. Many of the symbols used in Eqs. (2.1), (2.2), and (2.3) are listed below:

$N$ total number of particles in the whole beam,

$B$ bunching factor or the ratio of average to peak current

$\chi_{e} \quad$ neutralization factor,

$r_{0} \quad$ classical radius of the beam particle,

$R$ radius of the accelerator ring,

$\gamma, \beta$ relativistic factors of the beam particles,

$\nu_{0}^{V, H} \quad$ unperturbed vertical/horizontal betatron tune,

$h$ half vertical height of the vacuum chamber,

$h_{m} \quad$ half vertical gap between magnet pole faces,

$\mathcal{F}$ fraction of vacuum chamber with magnet pole faces,

$\epsilon_{1}^{V, H} \quad$ vertical/horizontal incoherent Laslett electric image coefficient,

$\epsilon_{2}^{V, H} \quad$ vertical/horizontal incoherent Laslett magnetic image coefficient,

$\xi_{1}^{V, H} \quad$ vertical/horizontal coherent Laslett electric image coefficient,

$\xi_{2}^{V, H} \quad$ vertical/horizontal coherent Laslett magnetic image coefficient,

$\epsilon_{\mathrm{spch}}^{V, H} \quad$ vertical/horizontal self-field coefficient,

$a_{V, H} \quad$ vertical/horizontal half size of the particle beam.

${ }^{\dagger}$ The ac magnetic field generated by betatron oscillations has frequency equal to the nonintegral part of the tune multiplied by the revolution frequency. For illustration, notice that a beam having an integer betatron tune is just a stationary ribbon inside the vacuum chamber with gaps due to bunching. At any fixed point along the ring, the field from the betatron motion therefore has zero frequency. 


\section{COMMENTS}

1. The tune shifts for a coasting beam can be obtained from the above expressions by letting the bunching factor $B$ equal unity.

2. Attention should be paid that the image coefficients are so defined that only the half height $h$ of the vacuum chamber or the vertical half magnetic pole gap $h_{m}$ appears in the tune-shift expressions, independent of whether we are dealing with the vertical tune shifts or horizontal tune shifts.

3. Many authors replace the unperturbed betatron tune $\nu_{0}^{V, H}$ with $R /\left\langle\beta^{V, H}\right\rangle$, where $\left\langle\beta^{V, H}\right\rangle$ is the average vertical/horizontal betatron function of the accelerator ring. Actually, it is more accurate to use $\nu_{0}^{V, H}$ because it comes from the perturbed equation of motion as $\left(\nu^{V, H}\right)^{2} \approx\left(\nu_{0}^{V, H}\right)^{2}+2 \nu_{0}^{V, H} \Delta \nu^{V, H}$. In fact, when the tune shift is large, $\Delta \nu^{V, H}$ on the left side of Eqs. (2.1) to (2.3) should be replaced by $\Delta\left(\nu^{V, H}\right)^{2} /\left(2 \nu_{0}^{V, H}\right)$.

4. Notice that there is a negative sign in front of each of the tune-shift expressions. This implies that a positive image or space-charge coefficient will contribute a downward shifting to the betatron tune.

5. The tune shifts we are discussing in Eqs. (2.1) to (2.3) and in the rest of this paper are all dipole tune shifts, because only the linear part of the transverse force has been included [see Eqs. (A.1) and (A.2)].

\section{SPACE-CHARGE COEFFICIENTS}

The space-charge tune shifts arise from the electromagnetic force acting on the test particle from all the particles in the beam without consideration of the presence of the vacuum chamber. For a beam of elliptical cross section with horizontal and vertical radii, $a_{H}$ and $a_{V}$, and of uniform distribution transversely, the self-force leads to the space-charge tune shifts

$$
\Delta \nu_{\text {spch incoh }}^{V, H}=-\frac{N r_{0} R}{\pi \gamma^{3} \beta^{2} B a_{V, H}\left(a_{V}+a_{H}\right) \nu_{0}^{V, H}},
$$


where we assume no particles of opposite charge are present or the neutralization factor $\chi_{e}=0$. These shifts are obviously incoherent. On the other hand, the (dipole) coherent space-charge tune shifts in both transverse directions are zero. This is understandable, because the center of the beam does not see its own space-charge force. The self-field or space-charge coefficients $\epsilon_{\text {spch }}^{V, H}$ in the vertical and horizontal directions are defined by the last term of Eq. (2.1). The space-charge coefficients take care of the transverse shape of the beam and how the beam particles are distributed. For a uniformly distributed beam, the space-charge tune shifts for each beam particle in a cross sectional slice are the same. The space-charge coefficients are

$$
\epsilon_{\mathrm{spch}}^{V}=\frac{a_{V}}{a_{V}+a_{H}} \quad \text { and } \quad \epsilon_{\mathrm{spch}}^{H}=\frac{a_{V}^{2}}{a_{H}\left(a_{V}+a_{H}\right)},
$$

which become $\frac{1}{2}$ when $a_{V}=a_{H}$.

For a beam with bi-Gaussian distribution in the transverse planes, theoretically there is no transverse beam boundary. In order to define the space-charge coefficient as in Eq. (2.1), we introduce the $95 \%$ beam size $a_{V, H}=\sqrt{6} \sigma_{V, H}$, where $\sigma_{V, H}$ represents the vertical/horizontal rms beam size. The space-charge coefficients are then

$$
\epsilon_{\mathrm{spch}}^{V}=\frac{f \sigma_{V}}{\sigma_{V}+\sigma_{H}} \quad \text { and } \quad \epsilon_{\mathrm{spch}}^{H}=\frac{f \sigma_{V}^{2}}{\sigma_{H}\left(\sigma_{V}+\sigma_{H}\right)},
$$

where the form factor $f$ comes about because each particle in a transverse slice of the beam receives different tune shifts. If we consider only the particles at the center of the beam where the tune shifts are largest, $f=3$. However, if we make a rough estimate by assuming those particles within one sigma of the beam core to have the maximum tune shifts while those outside do not experience any space-charge force, we obtain some sort of average for the particles in the cross sectional slice, $f=3\left(1-e^{-1 / 2}\right)=1.180$, which is only slightly larger than that for a uniformly distributed beam.

It is important to point out that what we really care for is the spread in space-charge tune shift among the particles inside the beam, but not so much the maximum spacecharge tune shift, because the latter can be corrected by changing the bare tune of the machine. For a distribution of finite extent, the space-charge tune spread is always less than the maximum space-charge tune spread, which occurs at the center of the beam for most distributions. For a transverse bi-Gaussian distribution that extend to infinity, the space-charge tune shift of a particle infinitely far away from the beam axis is zero, and 
therefore the space-charge tune spread is equal to the maximum space-charge tune shift. However, these particles are excluded from a realistic distribution which has a finite extent and this makes the space-charge tune spread less than the maximum tune shift. When the bi-Gaussian distribution is truncated more and more (by including only those particles closer and closer to the beam center), the space-charge tune spread becomes smaller and smaller while the maximum space-charge tune shift remains unchanged. For a round beam, with bi-Gaussian distribution, $\sigma_{r}=\sigma_{H}=\sigma_{V}$ and the form factor $f(r)$ in Eq. (3.3) for betatron amplitude $r$ is found to be

$$
\frac{f(r)}{3}=\frac{8}{\pi} \int_{0}^{\pi / 2}\left[1-\exp \left(-\frac{r^{2}}{\sigma_{r}^{2}} \sin ^{2} \theta\right)\right] d \theta=\sum_{n=1}^{\infty} \frac{(2 n) !}{2(n !)^{3}}\left(-\frac{r^{2}}{8 \sigma_{r}^{2}}\right)^{n-1}
$$

This is depicted in Fig. 1. Consider a beam with a bi-Gaussian distribution truncated at $2.5 \sigma_{r}$, we see that the particles at the edge of the beam have a space-charge tune shift

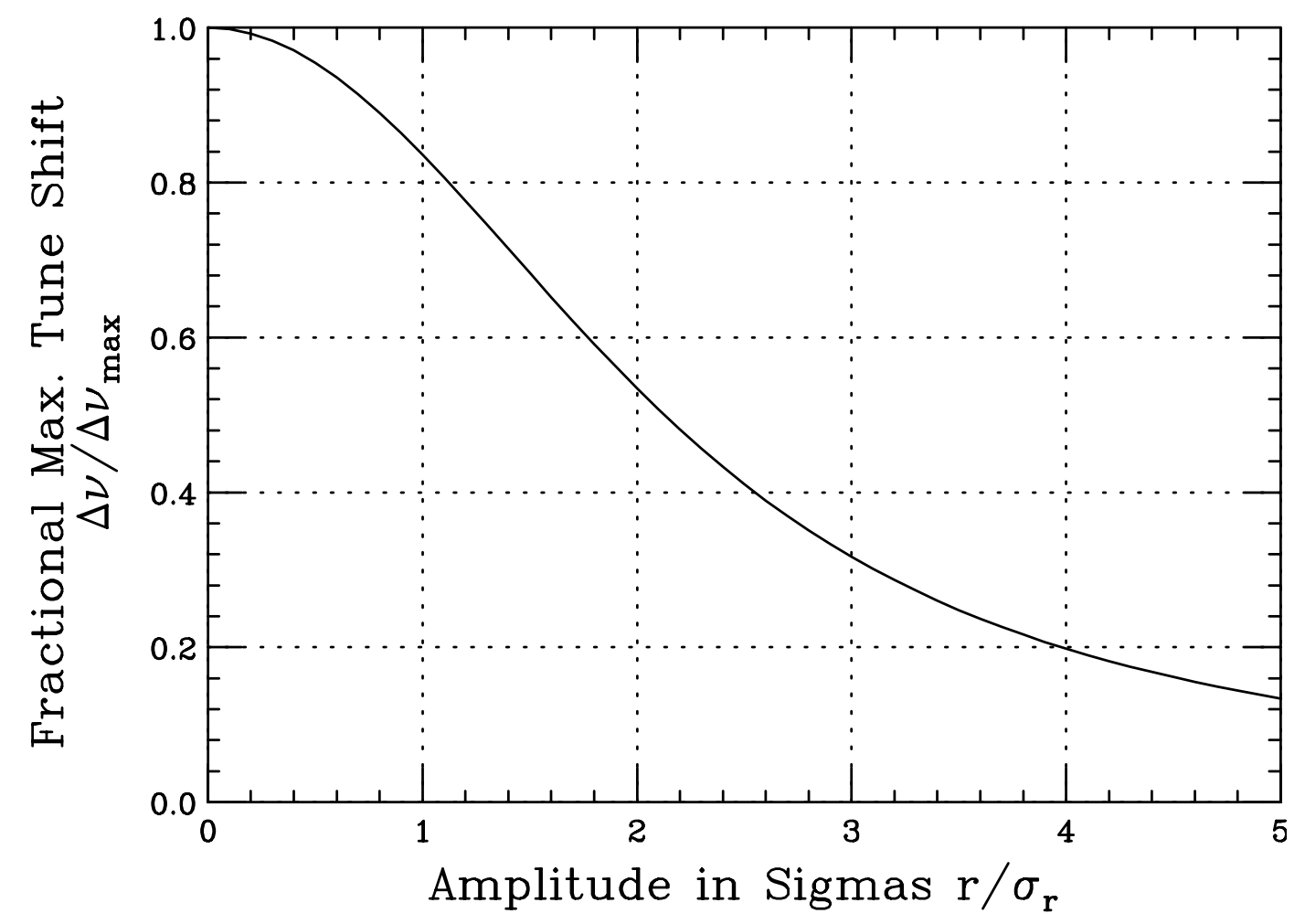

Figure 1: Plot of space-charge tune shift of a particle with betatron amplitude $r$ as a fraction of the maximum space-charge tune shift of a bi-Gaussian distributed round beam with rms beam size $\sigma_{r}$. 
$\sim 60 \%$ of the maximum space-charge tune shift. Thus the space-charge tune spread is equal to $\sim 60 \%$ the maximum space-charge tune shift. On the other hand, for the uniform transverse distribution, the space-charge tune shift is amplitude independent and the spread is zero exactly.

\section{ELLIPTICAL VACUUM CHAMBER}

The elliptical cross section of the vacuum chamber has half width $w$ and half height $h<w$. They are also known as the major and minor radii. The focal points are on the horizontal axis at distance $e=\sqrt{w^{2}-h^{2}}$ from the center. Consider a line beam on the horizontal axis at distance $x$ from the center. When the beam is inside the focal points or $x<e$, the image coefficients are ${ }^{\dagger}$

$$
\begin{gathered}
\epsilon_{1}^{V}=-\epsilon_{1}^{H}=\frac{h^{2}}{12 W^{2}}\left[A\left(\frac{2 K}{\pi \mathrm{cndn}}\right)^{2}+\frac{6 K k^{\prime 2} x \mathrm{sn}}{\pi W \mathrm{cndn}}-\frac{4 e^{2}+5 x^{2}}{2 W^{2}}\right], \\
\xi_{1}^{V}=\frac{h^{2}}{4 W^{2}}\left[\left(\frac{2 K \mathrm{dn}}{\pi \mathrm{cn}}\right)^{2}+\frac{2 K k^{\prime 2} x \mathrm{sn}}{\pi W \mathrm{cndn}}-\frac{e^{2}+x^{2}}{W^{2}}\right], \\
\xi_{1}^{H}=-\frac{h^{2}}{4 W^{2}}\left[\left(1-k^{2} \mathrm{sn}^{4}\right)\left(\frac{2 K k^{\prime}}{\pi \mathrm{cndn}}\right)^{2}+\frac{2 K k^{\prime 2} x \mathrm{sn}}{\pi W \mathrm{cndn}}-\frac{e^{2}+x^{2}}{W^{2}}\right],
\end{gathered}
$$

where

$$
A=\left(2-k^{2}\right)-\frac{1}{2}\left(1+k^{2}\right)^{2} \mathrm{sn}^{2}-k^{2}\left(1-2 k^{2}\right) \mathrm{sn}^{4}
$$

and

$$
W^{2}=e^{2}-x^{2}=w^{2}-h^{2}-x^{2} .
$$

The arguments of the Jacobian elliptic functions sn, cn, dn are

$$
\left(\frac{2 K(k)}{\pi} \sin ^{-1}(x / e), k\right)
$$

\footnotetext{
${ }^{\dagger}$ In Ref. [3], Table II, Part (c), the expression for $\epsilon_{1}$ when $e \leq x<W$, has a positive sign in front of the 1 , the last term inside the square brackets. The sign should have been negative. These expressions are also presented from Eqs. (74) to (76) in Ref. [2]. The expression following Eq. (74) is incorrect that the factor $\left(1+k^{2}+k^{4}\right)$ in the middle term should have been $\left(1+2 k^{2}+k^{4}\right)$. The first factor after the opening square bracket $\left(1-k^{2} S^{2}\right)$ should have been $\left(1-k^{2} S^{4}\right)$.
} 
where $K=K(k)$ is the complete elliptical function of the first kind and $k$ is called the modulus. ${ }^{\ddagger}$ The complementary modulus $k^{\prime}$ is given by

$$
k^{\prime}=\sqrt{1-k^{2}} .
$$

We first compute the nome, defined as

$$
q=\exp \left[-\frac{\pi K^{\prime}(k)}{K(k)}\right]
$$

using the expression

$$
q=\frac{w-h}{w+h},
$$

then the complementary modulus $k^{\prime}$ using $^{\S}$

$$
k^{\prime \frac{1}{2}}=\frac{1+2 \sum_{s=1}^{\infty}(-1)^{s} q^{s^{2}}}{1+2 \sum_{s=1}^{\infty} q^{s^{2}}},
$$

and finally the modulus $k$ through Eq. (4.7).

Notice that each term in Eqs. (4.1), (4.2), and (4.3) becomes singular when the beam approaches the focal points of the elliptic cross section. However, the singularities cancel each other in each expression to arrive at a finite value as $x \rightarrow e$. For this reason double precision must be used in evaluating these expressions. Right at the focal points the image coefficients become ${ }^{\top}$

$$
\begin{gathered}
\epsilon_{1}^{V}=-\epsilon_{1}^{H}=\frac{h^{2}}{360 e^{2}}\left[\left(1-16 k^{2}+k^{4}\right)\left(\frac{2 K}{\pi}\right)^{4}+10\left(1+k^{2}\right)\left(\frac{2 K}{\pi}\right)^{2}-11\right] \\
\xi_{1}^{V}=\frac{h^{2}}{180 e^{2}}\left[\left(2+13 k^{2}+2 k^{4}\right)\left(\frac{2 K}{\pi}\right)^{4}+5\left(1+k^{2}\right)\left(\frac{2 K}{\pi}\right)^{2}-7\right],
\end{gathered}
$$

${ }^{\ddagger}$ Some authors also define the parameter $m=k^{2}$ and the complementary parameter $m^{\prime}=k^{\prime 2}=1-m$.

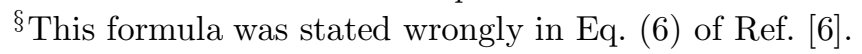

ף in Ref. [2], in Appendix D(f), the first term of $\xi_{1}^{V}$ was $\left(2-13 k^{2}+2 k^{4}\right)$ which has a wrong sign preceding $13 k^{2}$ as compared with our Eq. (4.12). In Ref. [3], Table II, Part (c), the expression for $\epsilon_{1}$ when $x=e$, has an overall incorrect sign. 


$$
\xi_{1}^{H}=\frac{-h^{2}}{180 e^{2}}\left[2\left(1-16 k^{2}+k^{4}\right)\left(\frac{2 K}{\pi}\right)^{4}+5\left(1+k^{2}\right)\left(\frac{2 K}{\pi}\right)^{2}-7\right] .
$$

When the beam is outside the focal points or $x>e$, the image coefficients assume the form ${ }^{\|}$

$$
\begin{gathered}
\epsilon_{1}^{V}=-\epsilon_{1}^{H}=\frac{h^{2}}{12 W^{2}}\left[B_{1}\left(\frac{2 K}{\pi \mathrm{sncn}}\right)^{2}+\frac{6 K x \mathrm{dn}}{\pi W \mathrm{sncn}}-\frac{4 e^{2}+5 x^{2}}{2 W^{2}}\right], \\
\xi_{1}^{V}=\frac{h^{2}}{4 W^{2}}\left[\left(\frac{2 K \mathrm{cn}}{\pi \mathrm{sn}}\right)^{2}+\frac{2 K x \mathrm{dn}}{\pi W \mathrm{sncn}}-\frac{e^{2}+x^{2}}{W^{2}}\right] \\
\xi_{1}^{H}=-\frac{h^{2}}{4 W^{2}}\left[B_{2}\left(\frac{2 K}{\pi \mathrm{sncn}}\right)^{2}+\frac{2 K x \mathrm{dn}}{\pi W \mathrm{sncn}}-\frac{e^{2}+x^{2}}{W^{2}}\right]
\end{gathered}
$$

where

$$
B_{1}=\frac{3}{2}-\frac{1}{2}\left(8-k^{\prime 2}\right) \mathrm{sn}^{2}+\left(1+k^{\prime 2}\right) \mathrm{sn}^{4}, \quad B_{2}=1-2 \mathrm{sn}^{2}+k^{\prime 2} \mathrm{sn}^{4}
$$

Unlike the situation when the beam is inside the focal points, here

$$
W^{2}=x^{2}-e^{2}=x^{2}-w^{2}+h^{2},
$$

and the Jacobian elliptic functions sn, cn, and dn have arguments

$$
\left(\frac{2 K(k)}{\pi} \cosh ^{-1}(x / e), k^{\prime}\right) \text {. }
$$

However, the nome $q$, modulus $k$, and complementary modulus $k^{\prime}$ are the same as given by Eqs. (4.9), (4.7), and (4.10).

\subsection{CENTERED BEAM}

When the beam is right at the center of the vacuum chamber, $x=0$. The arguments of the elliptic functions in Eq. (4.6) simplify to $(0, k)$ and we have $\mathrm{sn}=0, \mathrm{cn}=\mathrm{dn}=1$.

"In Ref. [2], Appendix D(e), the expressions for $\epsilon_{1}^{V}, \xi_{1}^{V}$, and $\xi_{1}^{H}$ have negative sign in front of the middle terms inside the squared brackets. They should be positive as given by Eqs. (4.14), (4.15), and (4.16). The expression for $B_{1}$ in Ref. [2] has the typo that $S$ in the second term on the right side should have been $S^{2}$. 
The expressions for the image coefficients in Eqs. (4.1), (4.2), and (4.3) simplify readily to

$$
\begin{gathered}
\epsilon_{1}^{V}=-\epsilon_{1}^{H}=\frac{h^{2}}{12 e^{2}}\left[\left(1+k^{\prime 2}\right)\left(\frac{2 K}{\pi}\right)^{2}-2\right], \\
\xi_{1}^{V}=\frac{h^{2}}{4 e^{2}}\left[\left(\frac{2 K}{\pi}\right)^{2}-1\right], \\
\xi_{1}^{H}=\frac{h^{2}}{4 e^{2}}\left[1-\left(\frac{2 K k^{\prime}}{\pi}\right)^{2}\right] .
\end{gathered}
$$

\section{CIRCULAR VACUUM CHAMBER}

When the ratio of minor to major radii $h / w=1$, the elliptic cross section becomes circular. Unfortunately, it is not easy to make the expressions of image coefficients for the elliptic cross section approach those for the circular cross section. This is because as $h / w \rightarrow 1$, the focal points move towards the center of the circle or $e \rightarrow 0$, making many terms in the elliptical formulas singular.

Fortunately, the image coefficients can be easily derived using the method of images. For a beam with fractional offset $g=x / r$, where $r$ is the radius of the circular cross section, the image coefficients are

$$
\begin{gathered}
\epsilon_{1}^{V}=-\epsilon_{1}^{H}=-\frac{g^{2}}{2\left(1-g^{2}\right)^{2}}, \\
\xi_{1}^{V}=\frac{1}{2\left(1-g^{2}\right)}, \\
\xi_{1}^{H}=\frac{1+g^{2}}{2\left(1-g^{2}\right)^{2}} .
\end{gathered}
$$

The transverse space-charge impedance of a beam in a vacuum chamber is given by

$$
Z_{1}^{V, H}=-\frac{i 2 R Z_{0}}{\beta^{2} \gamma^{2}}\left[\frac{1}{a_{V, H}\left(a_{V}+a_{H}\right)}-\frac{\xi_{1}^{V, H}-\epsilon_{1}^{V, H}}{h^{2}}\right],
$$

where $Z_{0} \approx 377 \Omega$ is the free-space impedance. Thus the combination $\xi_{1}^{V, H}-\epsilon_{1}^{V, H}$ is of 
importance. For the circular vacuum chamber, we have ${ }^{\dagger}$

$$
\xi_{1}^{V, H}-\epsilon_{1}^{V, H}=\frac{1}{2\left(1-g^{2}\right)^{2}} .
$$

When the beam is at the center of the circular vacuum chamber, Eqs. (5.1), (5.2), and (5.3) readily reduce to

$$
\begin{gathered}
\epsilon_{1}^{V}=-\epsilon_{1}^{H}=0 \\
\xi_{1}^{V}=\xi_{1}^{H}=\frac{1}{2}
\end{gathered}
$$

as expected from symmetry.

\section{RECTANGULAR VACUUM CHAMBER}

\subsection{OFF-CENTERED BEAM}

To conform with the elliptical beam pipe, let $h$ and $w$ be, respectively, the half height and half width of the rectangular cross section. ${ }^{\ddagger}$ When the beam is on the horizontal axis but with fractional offset $g$ (or at distance $g w$ from the center), the image coefficients $\operatorname{are}^{\S}$

$$
\begin{gathered}
\epsilon_{1}^{V}=-\epsilon_{1}^{H}=\frac{K^{2}(k)}{4}\left[\frac{k^{\prime 4} \mathrm{sn}^{2} \mathrm{cn}^{2}}{2 \mathrm{dn}^{2}}-\frac{k^{\prime 2}\left(1-2 \mathrm{sn}^{2}\right)}{3}-\frac{\mathrm{dn}^{2}\left(3-4 \mathrm{sn}^{2}+4 \mathrm{sn}^{4}\right)}{6 \mathrm{sn}^{2} \mathrm{cn}^{2}}\right], \\
\xi_{1}^{V}=\frac{K^{2}(k)}{4} \frac{k^{\prime 4} \mathrm{sn}^{2} \mathrm{cn}^{2}}{\mathrm{dn}^{2}} \\
\xi_{1}^{H}=\frac{K^{2}(k)}{4}\left[k^{\prime 2}\left(1-2 \mathrm{sn}^{2}\right)+\frac{\mathrm{dn}^{2}}{\mathrm{sn}^{2} \mathrm{cn}^{2}}\right] .
\end{gathered}
$$

The arguments of the elliptic functions sn, cn, dn are

$$
\left(\frac{K(k) y_{0}}{h}, k^{\prime}\right)=\left(\frac{K(k) w}{h}(1-g), k^{\prime}\right)
$$

where $y_{0}=(1-g) w$ is the position of the beam measured from one vertical wall of the vacuum chamber, and $K(k)$ is the complete elliptical function of the first kind.

\footnotetext{
${ }^{\dagger}$ In Ref. [6], the equation after Eq. (61) is incorrect by first an overall negative sign and second having $g^{2}$ written wrongly as $g$. Also in Fig. 5, the ordinate should have been $\xi_{1}^{V, H}-\epsilon_{1}^{V, H}$.

${ }^{\ddagger}$ Note that in Ref. [6], $h$ and $w$ are the full height and full width of the rectangular cross section.

${ }^{\S}$ Equation (6.1) was reported in Eq. (53) of Ref. [6] with a wrong sign in front of $\mathrm{sn}_{10}^{4}$ inside the last term in the curly brackets.
} 
Here, the nome is computed according to

$$
q=e^{-2 \pi w / h},
$$

which is quite different from the one used in the elliptical beam pipe [Eq. (4.9)]. Next, the complementary modulus $k^{\prime}$ can be computed from Eq. (4.10), from which the modulus $k$ can be obtained via Eq. (4.7).

\subsection{CENTERED BEAM}

For a centered beam, $g=0$, the arguments of the elliptical functions become

$$
\left(\frac{K(k) w}{h}, k^{\prime}\right)=\left(\frac{1}{2} K^{\prime}(k), k^{\prime}\right)=\left(\frac{1}{2} K\left(k^{\prime}\right), k^{\prime}\right) .
$$

Notice that the periods of sn, cn, dn with modulus $k^{\prime}$ are $4 K\left(k^{\prime}\right)$. The elliptical functions simplify to [5]

$$
\operatorname{sn}\left(\frac{1}{2} K\left(k^{\prime}\right), k^{\prime}\right)=\frac{1}{\sqrt{1+k}}, \quad \operatorname{cn}\left(\frac{1}{2} K\left(k^{\prime}\right), k^{\prime}\right)=\frac{\sqrt{k}}{\sqrt{1+k}}, \quad \operatorname{dn}\left(\frac{1}{2} K\left(k^{\prime}\right), k^{\prime}\right)=\sqrt{k} .
$$

The electric image coefficients simplify to

$$
\begin{gathered}
\epsilon_{1}^{V}=-\epsilon_{1}^{H}=\frac{K^{2}(k)}{12}\left(1-6 k+k^{2}\right), \\
\xi_{1}^{V}=\frac{K^{2}(k)}{4}(1-k)^{2}, \\
\xi_{1}^{H}=K^{2}(k) k,
\end{gathered}
$$

which involve only the complete elliptical function of the first kind.

\section{COMMENTS}

1. Since $q$ decreases exponentially as $w / h$ increases, very accurate value of $k^{\prime}$ can be computed with Eq. (4.10). For example, even for $1 \geq w / h \geq 0.2$, 14-figure accuracy can be readily obtained for $k^{\prime}$ and also $k^{2}$ afterward using Eq. (4.7), when the summations are extended to $s=5$. In fact, for centered beam, there is no need to go to $w / h<1$, because we can interchange the role of $w$ and $h$. 
2. When $w / k>1, q$ becomes very small and $k^{\prime}$ is very close to 1 . (For example, $k^{2}=2.9437 \times 10^{-3}, 5.5796 \times 10^{-5}$ and $1.0420 \times 10^{-7}$, respectively, when $w / h=1$, 2 and 3.) Equation (4.7) can no longer give accurate result for $k$. To preserve accuracy, we must expand $k^{2}$ as power series in $q$ with the aid of Eqs. (4.7) and (4.10):

$$
k^{2}=16 q\left(1-8 q+44 q^{2}-192 q^{3}+718 q^{4}-2400 q^{5}+7352 q^{6}-20992 q^{7}+56549 q^{8}-\cdots\right),
$$

from which 14-figure accuracy can be obtained when $w / k \geq 1$.

3. Because $k^{2} \ll 1$ when $w / h>1$, Eqs. (6.8), (6.9), and (6.10) can be viewed as expansions from values for the infinite horizontal plates. In fact, with

$$
K(k)=\frac{\pi}{2}\left[1+\frac{1}{4} k^{2}+\frac{9}{64} k^{4}+\mathcal{O}\left(k^{6}\right)\right],
$$

we can write

$$
\begin{gathered}
\epsilon_{1}^{V}=-\epsilon_{1}^{H}=\frac{\pi^{2}}{48}\left[1-6 k+\frac{3}{2} k^{2}-3 k^{3}+\frac{27}{32} k^{4}-\frac{33}{16} k^{5}+\mathcal{O}\left(k^{6}\right)\right] \\
\xi_{1}^{V}=\frac{\pi^{2}}{16}\left[1-2 k+\frac{3}{2} k^{2}-k^{3}+\frac{27}{32} k^{4}-\frac{11}{16} k^{5}+\mathcal{O}\left(k^{6}\right)\right] \\
\xi_{1}^{H}=\frac{\pi^{2}}{4} k\left[1+\frac{1}{2} k^{2}+\frac{11}{32} k^{4}+\mathcal{O}\left(k^{6}\right)\right]
\end{gathered}
$$

\section{TWO INFINITE PARALLEL PLATES}

When the width to height $w / h$ of the rectangle goes to infinity, the cross section of the vacuum chamber becomes two infinite parallel plates. As $w / h \rightarrow \infty$, the nome $q \rightarrow 0$. Thus $k^{\prime} \rightarrow 1$ and $k \rightarrow 0$. The complete elliptical function becomes $K(k) \rightarrow \pi / 2$. Equations. (6.13), (6.14), and (6.15) become

$$
\epsilon_{1}^{V}=-\epsilon_{1}^{H}=\frac{\pi^{2}}{48}, \quad \xi_{1}^{V}=\frac{\pi^{2}}{16}, \quad \xi_{1}^{H}=0,
$$

which are just the electric image coefficients for a beam in the middle of two infinite horizontal plates. Needless to say, these coefficients can be derived much more easily 
using the method of images, which also provides us with the magnetic images

$$
\epsilon_{2}^{V}=-\epsilon_{2}^{H}=\frac{\pi^{2}}{24}, \quad \xi_{2}^{V}=\frac{\pi^{2}}{16}, \quad \xi_{2}^{H}=0 .
$$

These image coefficients are important because the magnet pole faces are often considered as two infinite parallel horizontal plates. Theoretically, these image coefficients can also be obtained from the image coefficients for elliptical cross section in the limit $h / w \rightarrow 0$. In practice, however, this reduction is far from trivial. This is because as $w / h \rightarrow \infty$, the nome $q \rightarrow 1$ according to Eq. (4.9) and we are not able to determine the complementary modulus $k^{\prime}$ from Eq. (4.10) by series expansion. However, the nome is defined mathematically as

$$
q=e^{-\pi K\left(k^{\prime}\right) / K(k)}
$$

which implies that $K(k) \rightarrow \infty$ and $k \rightarrow 1$. Take Eq. (4.20), for example, the squared brackets go to infinity while the eccentricity $e$ also goes to infinity. Thus the image coefficients cannot be determined easily as $w / h \rightarrow \infty$.

\section{PLOTS OF IMAGE COEFFICIENTS}

Actually the image coefficients for a centered beam in an elliptical or rectangular vacuum chamber depend on only one parameter, $h / w$, the ratio of the minor to major radii or the ratio of height to width. With an off-centered beam, there is another parameter, $g=x / w$, the fractional offset from the center of the vacuum chamber. The electric image coefficients for an elliptical vacuum chamber are plotted as functions of $g$ in Fig. 2, 3, and 4 for values of $h / w$ ranging from 0.1 to 1 . Notice that the ellipse becomes a circle when $h / w=1$. The electric image coefficients for a rectangular vacuum chamber are plotted in Figs. 5, 6, and 7 as functions of the beam offset $g$ from the center of the beam pipe, for aspect ratio from $h / w=0.1$ to 1.0. The rectangle becomes a square when $h / w=1$.

Although the behaviors for $\epsilon_{1}^{V \cdot H}$ and $\xi_{1}^{H}$ are similar for an offset beam inside an elliptical or rectangular vacuum chamber, the behaviors for $\xi_{1}^{V}$ are very much different. Consider a beam in a rectangular vacuum chamber which is very off-centered horizontally. When it is very close to one of the vertical walls, the beam would be seeing just an infinite vertical wall as if the other sides of the rectangular vacuum chamber were absent. This is illustrated in Fig. 8 with the beam at Point A and the image at Point A'. 
Table I: Self-field coefficients for uniformly charged beam and image coefficients for centered beam.

\begin{tabular}{|c|c|c|c|c|}
\hline Coeff. & Circular & Elliptical & Rectangular & $\begin{array}{l}\text { Parallel } \\
\text { Plates }\end{array}$ \\
\hline$\epsilon_{\mathrm{spch}}^{V}$ & $\frac{1}{2}$ & $\frac{a_{V}}{a_{H}+a_{V}}$ & & \\
\hline$\epsilon_{\mathrm{spch}}^{H}$ & $\frac{1}{2}$ & $\frac{a_{V}^{2}}{a_{H}\left(a_{H}+a_{V}\right)}$ & & \\
\hline$\epsilon_{1}^{V}$ & 0 & $\frac{h^{2}}{12 e^{2}}\left[\left(1+k^{\prime 2}\right)\left(\frac{2 K}{\pi}\right)^{2}-2\right]$ & $\frac{K^{2}(k)}{12}\left(1-6 k+k^{2}\right)$ & $\frac{\pi^{2}}{48}$ \\
\hline$\epsilon_{1}^{H}$ & 0 & $\frac{-h^{2}}{12 e^{2}}\left[\left(1+k^{\prime 2}\right)\left(\frac{2 K}{\pi}\right)^{2}-2\right]$ & $\frac{-K^{2}(k)}{12}\left(1-6 k+k^{2}\right)$ & $-\frac{\pi^{2}}{48}$ \\
\hline$\epsilon_{2}^{V}$ & $*$ & * & $*$ & $\frac{\pi^{2}}{24}$ \\
\hline$\epsilon_{2}^{H}$ & $*$ & $*$ & $*$ & $-\frac{\pi^{2}}{24}$ \\
\hline$\xi_{1}^{V}$ & $\frac{1}{2}$ & $\frac{h^{2}}{4 e^{2}}\left[\left(\frac{2 K}{\pi}\right)^{2}-1\right]$ & $\frac{K^{2}(k)}{4}(1-k)^{2}$ & $\frac{\pi^{2}}{16}$ \\
\hline$\xi_{1}^{H}$ & $\frac{1}{2}$ & $\frac{h^{2}}{4 e^{2}}\left[1-\left(\frac{2 K k^{\prime}}{\pi}\right)^{2}\right]$ & $K^{2}(k) k$ & 0 \\
\hline$\xi_{2}^{V}$ & $*$ & $*$ & $*$ & $\frac{\pi^{2}}{16}$ \\
\hline$\xi_{2}^{H}$ & $*$ & * & $*$ & 0 \\
\hline
\end{tabular}

* $\epsilon_{2}$ and $\xi_{2}$ for closed magnetic boundary (e.g., circular, elliptic, or rectangular) cannot be calculated when the relative permeability $\mu_{r} \rightarrow \infty$, since the induced magnetic field would not permit a charged beam to pass through because the field energy would become infinite. Closed magnetic yokes are used in superconducting magnets, but there the coefficients $\epsilon_{2}=\xi_{2} \rightarrow 0$, since the magnetic material is driven completely into saturation $\left(\mu_{r} \rightarrow 1\right)$.

$K(k)$ is the complete elliptic integral of the first kind. $k$ is determined from $(w-h) /(w+$ $h)=\exp \left(-\pi K^{\prime} / K\right)$ for the elliptical cross section but $w / h=K^{\prime} /(2 K)$ for the rectangular cross section, where $w$ and $h$ are the half width and half height, with $e=\sqrt{w^{2}-h^{2}}$, and $K^{\prime}=K\left(k^{\prime}\right)$ with $k^{\prime}=\sqrt{1-k^{2}}$. 


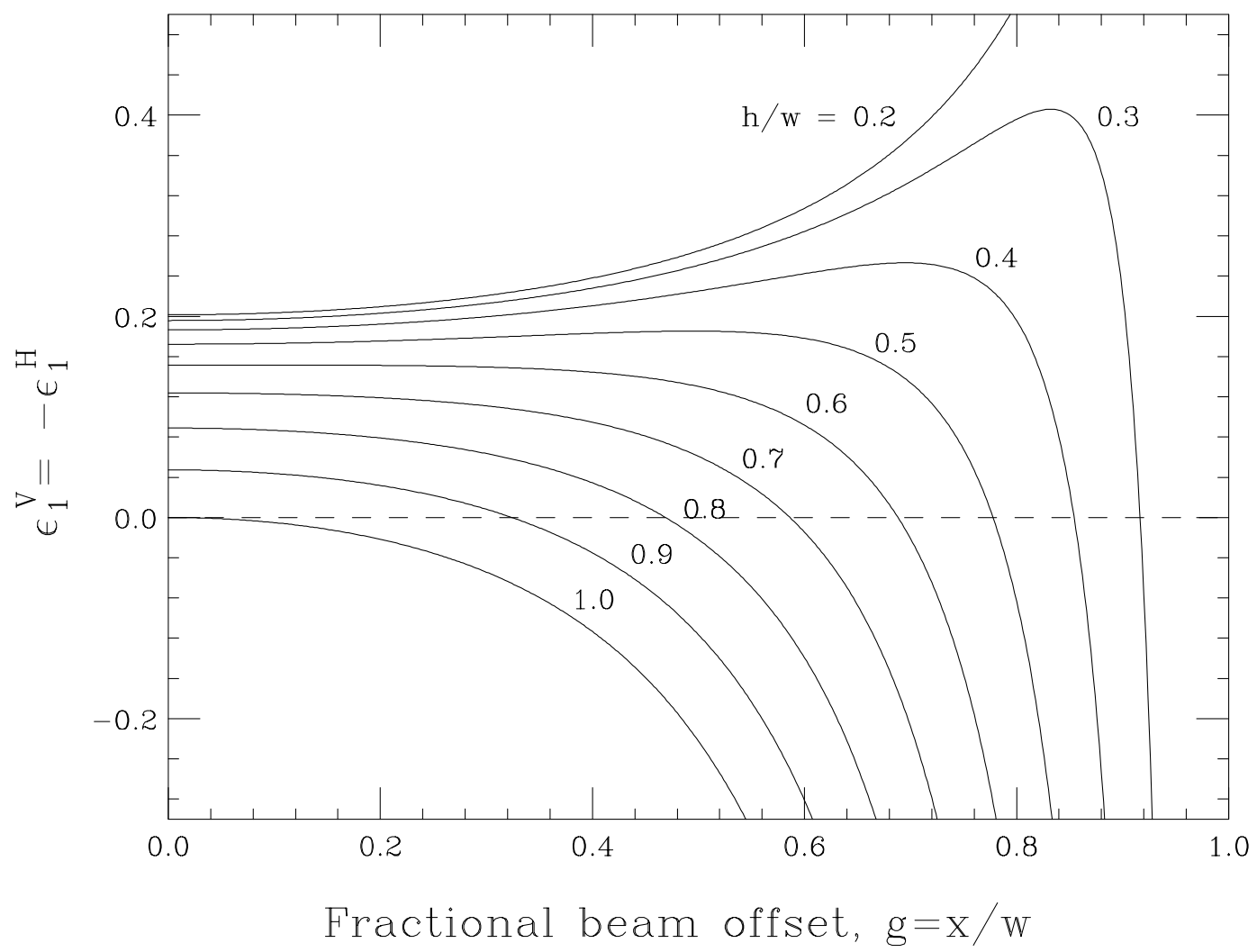

Figure 2: Plot of vertical (negative horizontal) coherent electric image coefficient $\epsilon_{1}^{V}=-\epsilon_{1}^{H}$ versus beam offset $g=x / w$ from the center of a vacuum chamber having an elliptical cross section of minor to major radii $h / w=0.2,0.3,0.4,0.5,0.6,0.7$, $0.8,0.9,1.0$. The situation of $h / w=1$ is equivalent to a vacuum chamber of circular cross section. The curve for $h / w=0.1$ has not been computed, because we have not been able to locate a library function for $K(k)$ that is capable to provide us with a value when $k$ is too close to unity. 


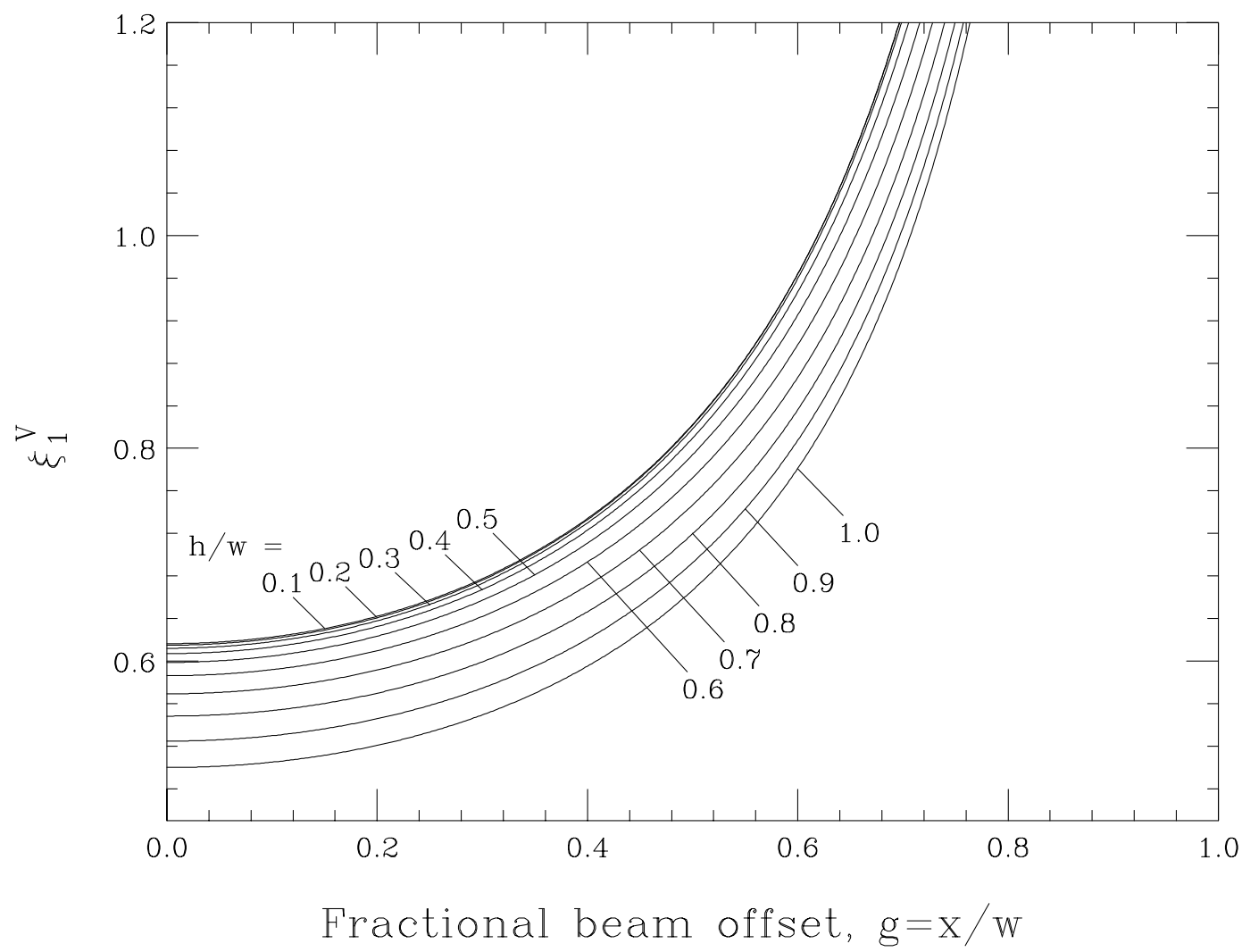

Figure 3: Plot of vertical coherent electric image coefficient $\xi_{1}^{V}$ versus beam offset $g=x / w$ from the center of a vacuum chamber having an elliptical cross section of minor to major radii $h / w=0.1,0.2,0.3,0.4,0.5,0.6,0.7,0.8,0.9,1.0$. The situation of $h / w=1$ is equivalent to a vacuum chamber of circular cross section. 


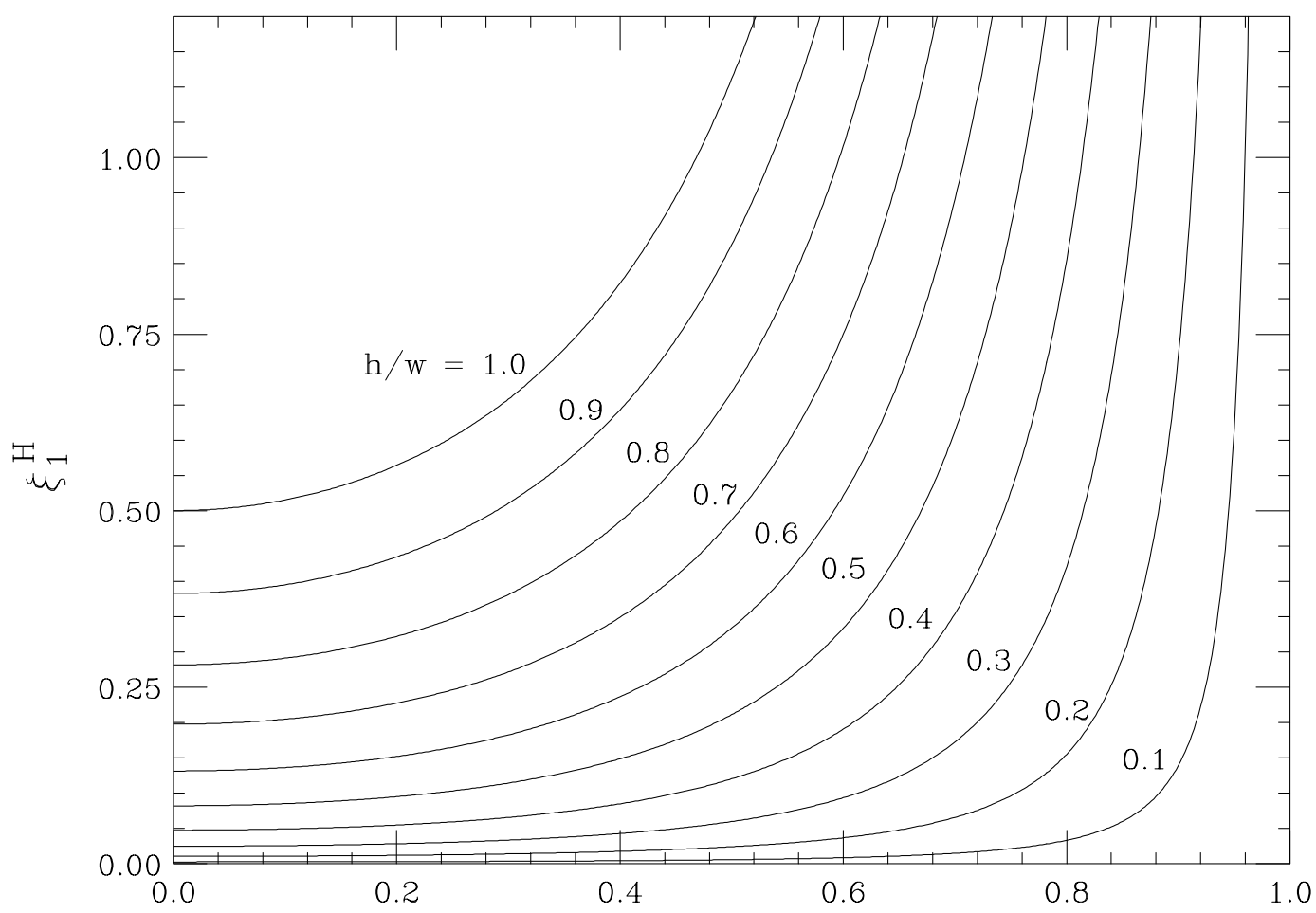

Fractional beam offset, $\mathrm{g}=\mathrm{x} / \mathrm{w}$

Figure 4: Plot of horizontal coherent electric image coefficient $\xi_{1}^{H}$ versus beam offset $g=x / w$ from the center of a vacuum chamber having an elliptical cross section of minor to major radii $h / w=0.1,0.2,0.3,0.4,0.5,0.6,0.7,0.8,0.9,1.0$. The situation of $h / w=1$ is equivalent to a vacuum chamber of circular cross section. 


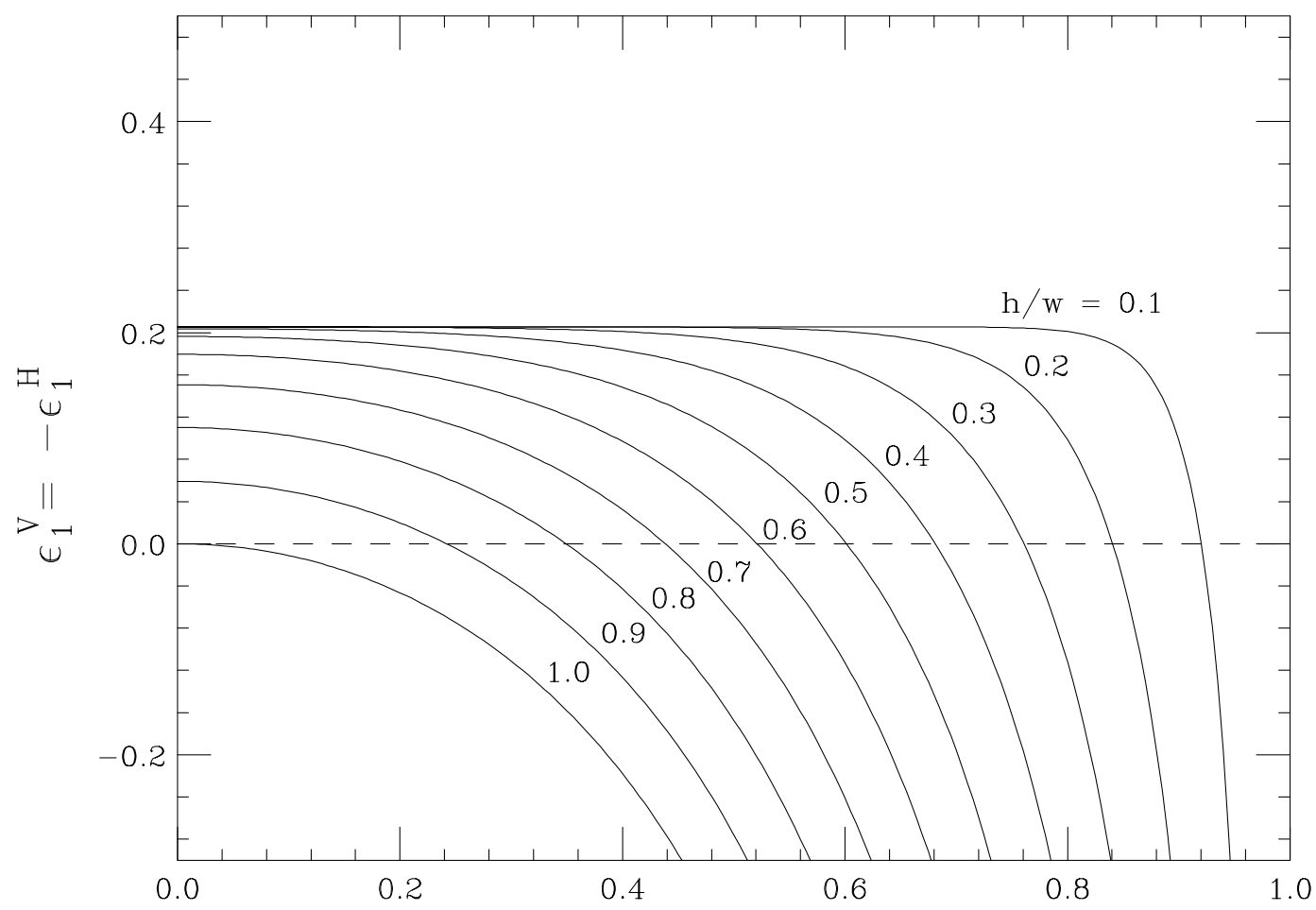

Fractional beam offset, $\mathrm{g}=\mathrm{x} / \mathrm{w}$

Figure 5: Plot of vertical (negative horizontal) coherent electric image coefficient $\epsilon_{1}^{V}=-\epsilon_{1}^{H}$ versus beam offset $g=x / w$ from the center of a vacuum chamber having a rectangular cross section of half-height to half-width $h / w=0.1,0.2,0.3,0.4,0.5$, $0.6,0.7,0.8,0.9,1.0$. The situation of $h / w=1$ is equivalent to a vacuum chamber of square cross section. 


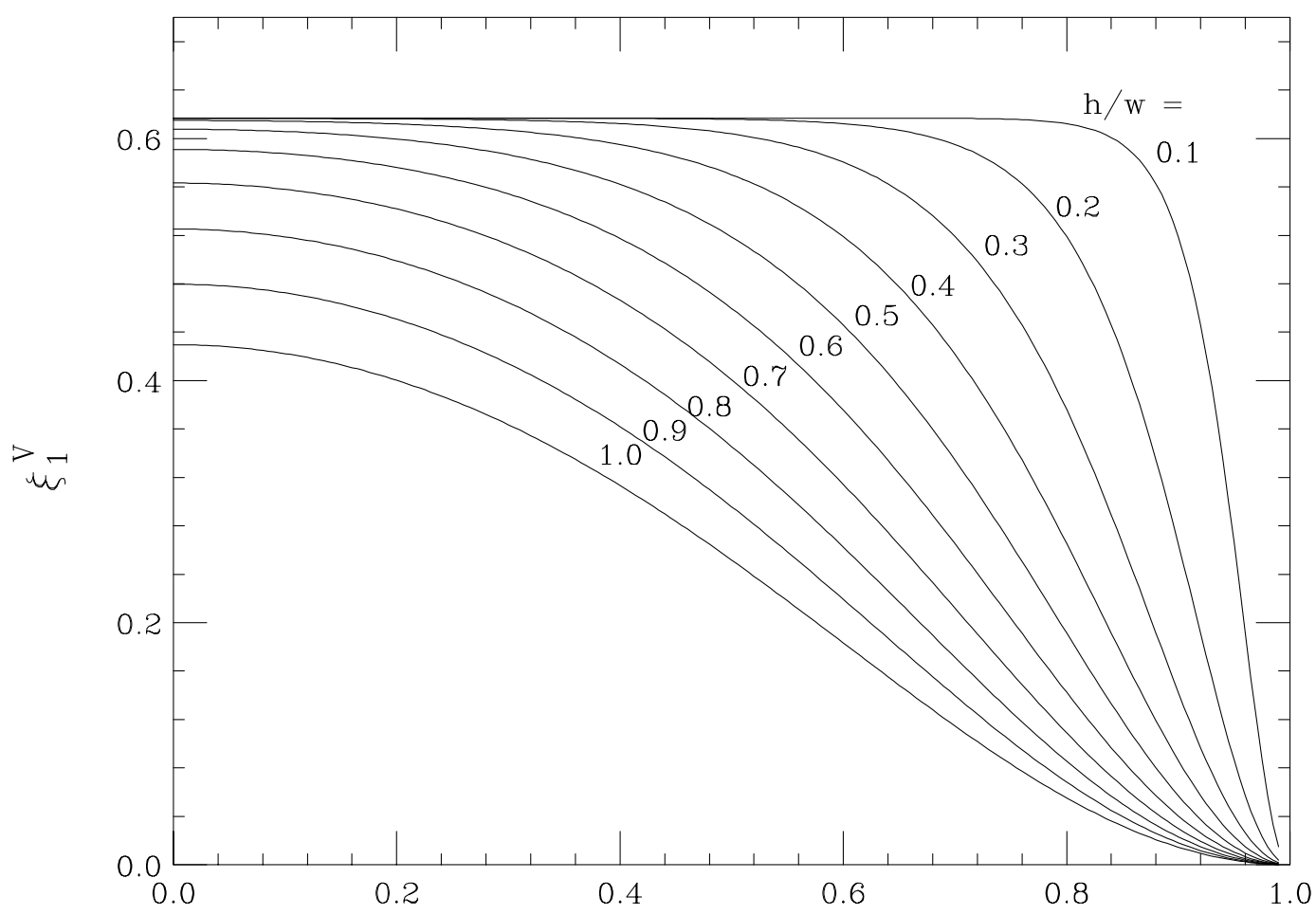

Fractional beam offset, $\mathrm{g}=\mathrm{x} / \mathrm{w}$

Figure 6: Plot of vertical coherent electric image coefficient $\xi_{1}^{V}$ versus beam offset $g=x / w$ from the center of a vacuum chamber having a rectangular cross section of half-height to half-width $h / w=0.1,0.2,0.3,0.4,0.5,0.6,0.7,0.8,0.9,1.0$. The situation of $h / w=1$ is equivalent to a vacuum chamber of square cross section. 


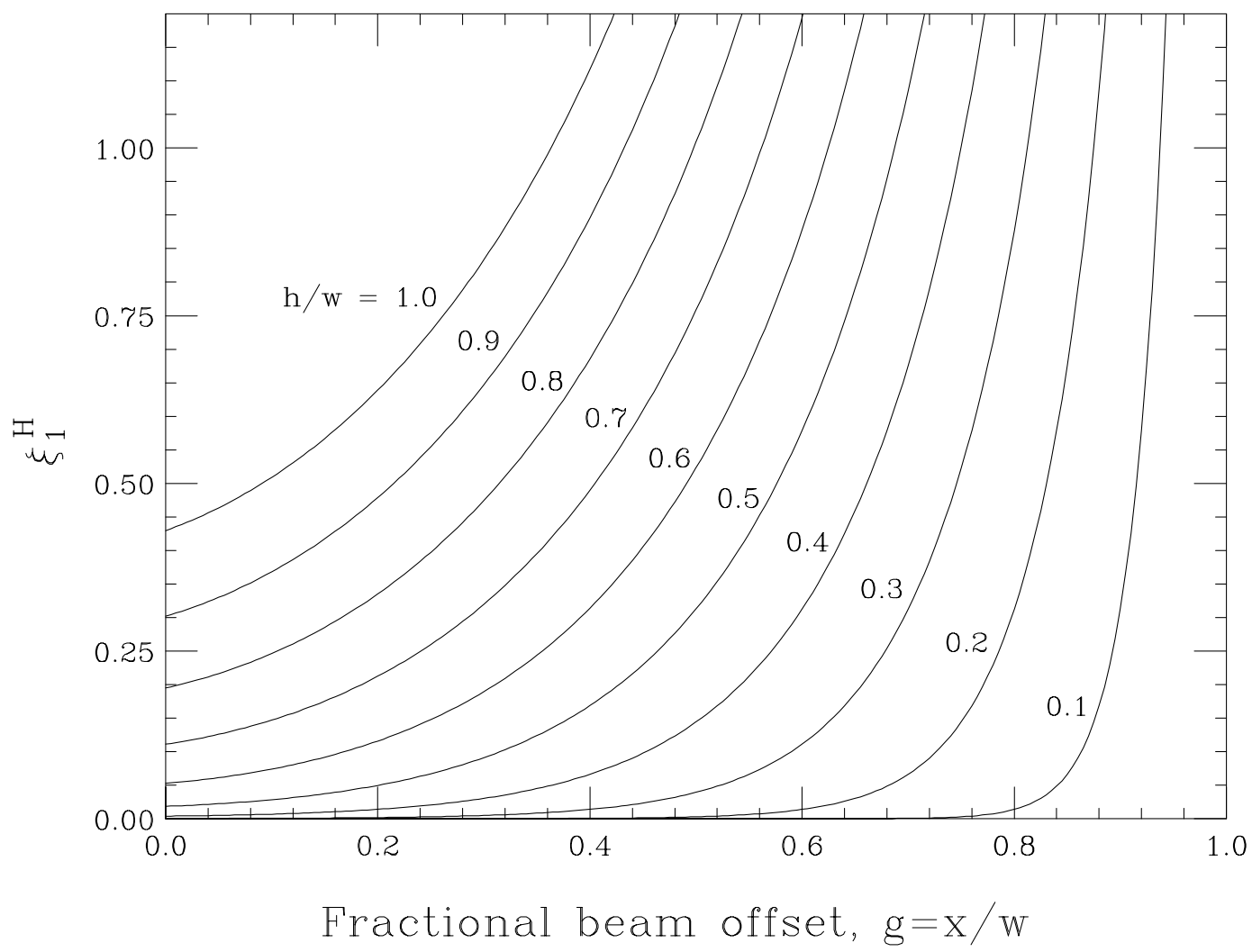

Figure 7: Plot of horizontal coherent electric image coefficient $\xi_{1}^{H}$ versus beam offset $g=x / w$ from the center of a vacuum chamber having a rectangular cross section of half-height to half-width $h / w=0.1,0.2,0.3,0.4,0.5,0.6,0.7,0.8,0.9,1.0$. The situation of $h / w=1$ is equivalent to a vacuum chamber of square cross section. 
The beam will therefore be affected by its only image in the vertical wall. When the beam moves vertically to Point B, the image moves with it to Point B'. Since the vertical displacements of the beam and image are always the same, the beam is seeing no vertical force from the image. The vertical coherent electric image coefficient $\xi_{1}^{V}$ must therefore vanish when the offset $x \rightarrow w$ or $g=x / w \rightarrow 1$, which is exactly what we see in Fig. 6. As for the horizontal motion, the image moves closer the the mean when the beam moves closer to the wall. The image force which is totally horizontal will lower the restoring force of the horizontal betatron oscillation. We therefore expect the horizontal coherent electric image coefficient $\xi_{1}^{H} \rightarrow \infty$ as the offset $g \rightarrow 1$, which is what we obtain in Fig. 7.

The situation is very different for an elliptical chamber. When the beam is so offcentered that it is near one of the horizontal edges, the beam does not see an infinite vertical plane as in the rectangular case. What it sees is a curved surface, like that of a circle. For simplicity, let us study the special case of a circular vacuum chamber. When the beam is at Point $\mathrm{A}$ on the horizontal axis at a distance $x$ from the center, the image is at Point A' also on the horizontal axis but at a distance

$$
x^{\prime}=\frac{r^{2}}{x},
$$

where $r$ is the radius of the circular vacuum chamber, as illustrated in Fig. 9. When the beam makes vertical betatron oscillations and is displaced vertically to Point $\mathrm{B}$ on the radius at angle $\theta$, the image also moves to Point $\mathrm{B}^{\prime}$ on the radius at angle $\theta$. Notice that Point B' is always higher than Point B. The image, being of opposite charge, will provide a vertical detuning force. As the beam is closer to the wall of the circular chamber, the beam at Point B and the image at Point B' come closer together and the vertical detuning force is increased. Therefore, the vertical coherent electric image coefficient $\xi_{1}^{V}$ will become larger when the beam is off-centered and reaches positive infinity as $g \rightarrow 1$, just as what we have plotted in Fig. 3. Horizontally, the image moves closer when the beam is closer to the circular wall of the chamber, thus providing a horizontal detuning force and the horizontal coherent electric image coefficient $\xi_{1}^{H} \rightarrow \infty$ as $g \rightarrow 1$, which is exactly what we see in Fig. 4 .

Now let us study the incoherent image force for a very much off-centered beam. For the rectangular vacuum chamber, the beam is again seeing its own image in an infinite vertical wall. Now the beam is stationary on the horizontal axis at Point A and the 


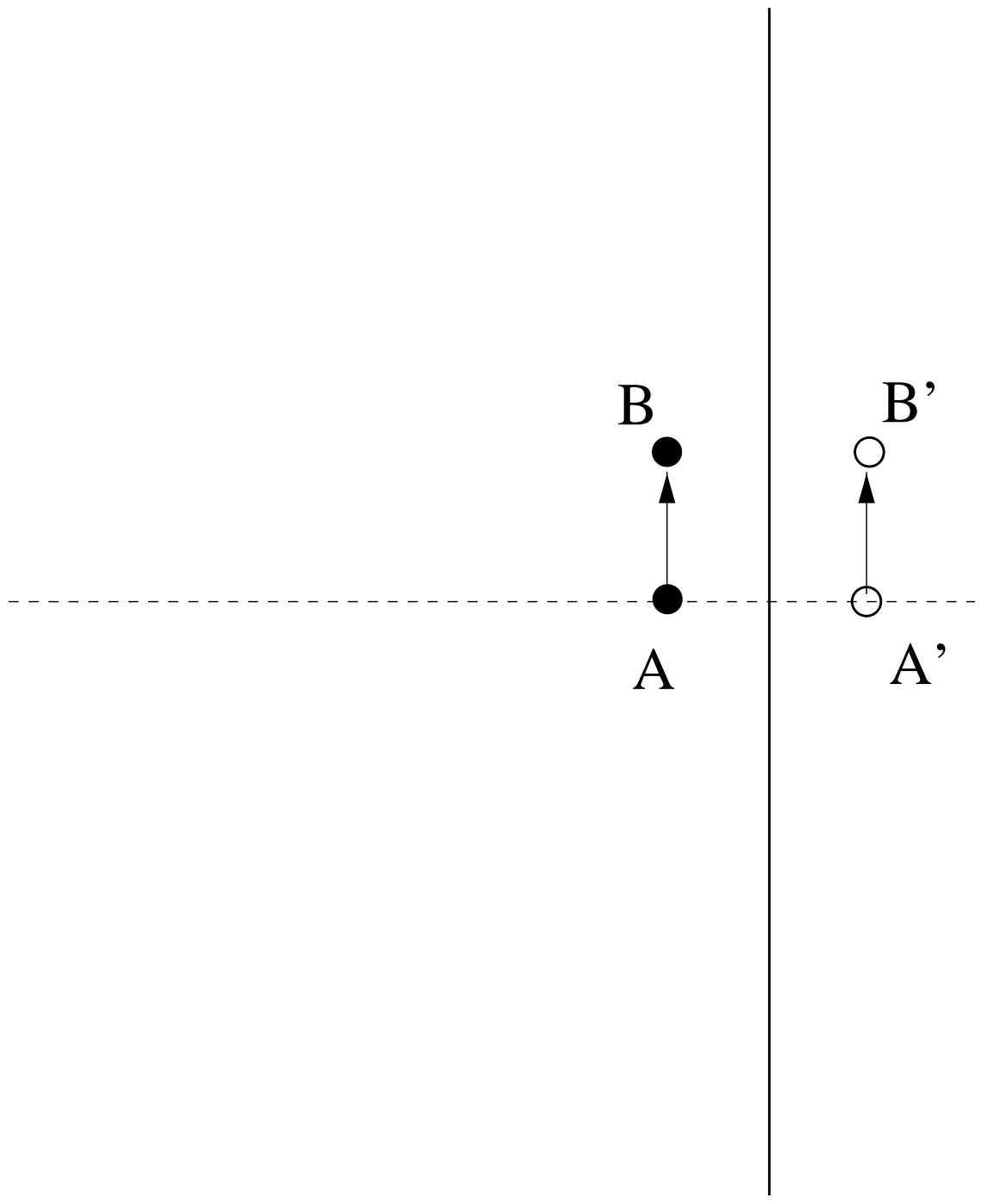

Figure 8: When the beam is on the horizontal axis of a rectangular vacuum chamber at Point A close to one of the vertical walls, the vacuum chamber can be approximated by an infinite vertical plane with the image at Point A'. As the beam is displaced vertically to Point $B$, the image moves to Point B' which has exactly the same vertical displacement as the beam, thus the image provides no vertical restoring force on the beam. 


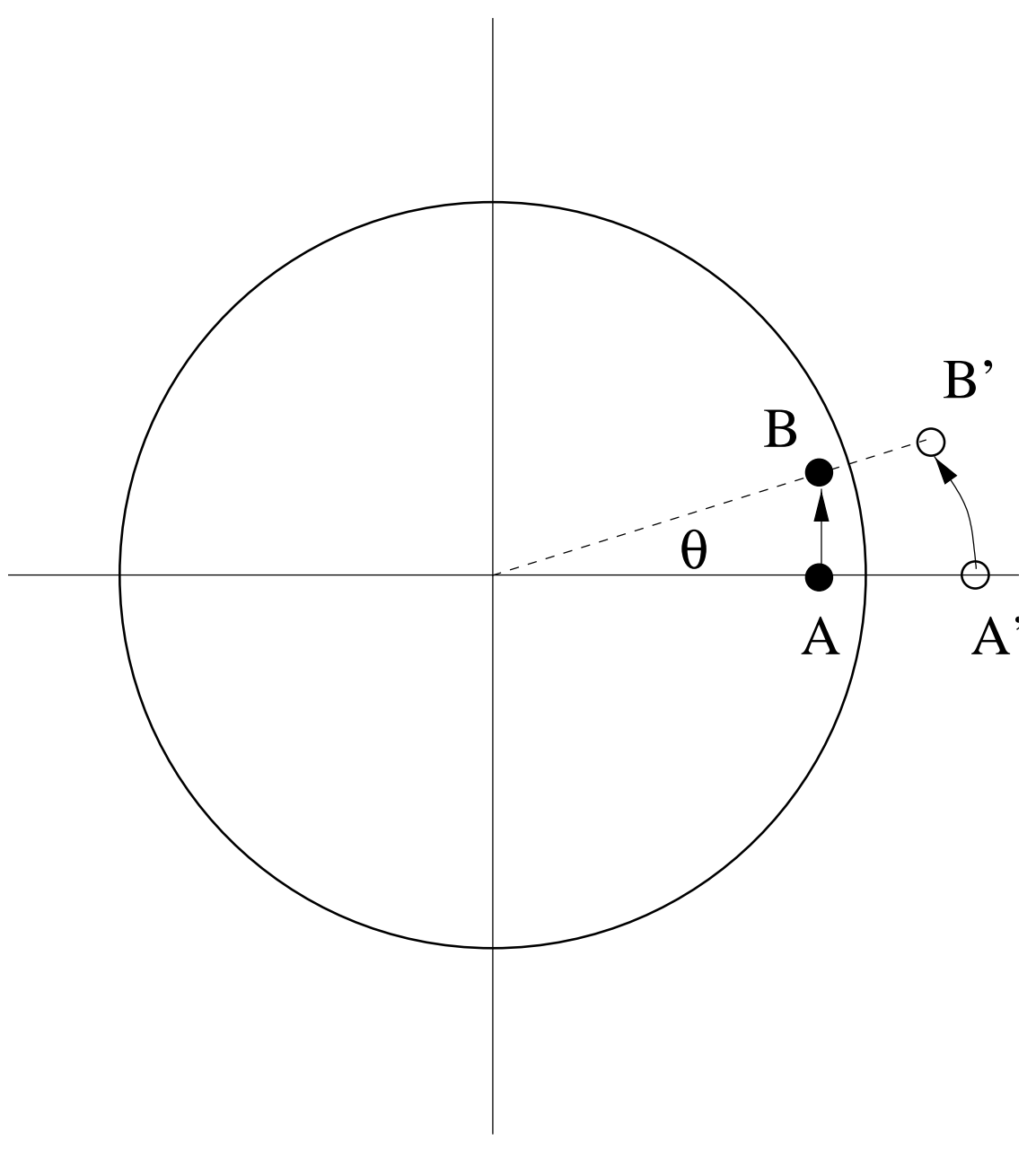

Figure 9: When the beam is on the horizontal axis of an elliptical vacuum chamber at Point A close to the wall, the vacuum chamber can be approximated as being circular with the image at Point A'. When the beam is displaced vertically to Point B, the image moves to Point B' which is always higher than the beam and therefore provides a coherent detuning to the vertical betatron oscillation. 
image at Point A' in Fig 8. Consider a test particle displaced vertically to Point B. It is attracted vertically downward by the beam image at Point A', thus enhancing the betatron restoring force. The enhancement increases as the offset is larger because the test particle will be closer to the beam image. We therefore expect to see the vertical incoherent electric image coefficient $\epsilon_{1}^{V}$ to drop gradually to negative infinity for an offset beam, as is plotted in Fig. 5. When the test particle is pushed horizontally from the stationary beam in the process of performing horizontal betatron oscillation, the image force increases in the outward direction as the beam offset is increased, thus providing a detuning and a smaller horizontal incoherent electric image coefficient $\epsilon_{1}^{H}$. The situation of the elliptic vacuum chamber is similar. Again both the beam and its image are stationary, respectively, at Point A and Point A' in Fig. 9. A test particle displaced vertically to Point $B$ will see an increasing restoring force from the beam image at Point A' when the beam offset is increased, so the vertical incoherent electric image coefficient $\epsilon_{1}^{V}$ decreases to negative infinity as the beam is offset more and more, as is plotted in Fig. 2.

To conclude this section, we compare the electric image coefficients for an elliptic vacuum chamber and a rectangular vacuum chamber when the beam is centered. We see from Fig. 10 that, independent of whether the cross section is elliptical or rectangular, the image coefficients $\epsilon_{1}^{V}, \xi_{1}^{V}$, and $\xi_{1}^{H}$ approach their correct values of $\pi^{2} / 48, \pi^{2} / 16$, and 0 for two parallel plates when $h / w \rightarrow 0$. However, when $h / w \rightarrow 1, \xi_{1}^{V}$ and $\xi_{1}^{H}$ approach 0.5 for the elliptic cross section but 0.430 for the rectangular cross section. This is understandable, because the former approaches a circle while the latter approaches a square. Some of the beam image current goes to the regions near the corners of the square and will not affect the beam so efficiently as in the case of the circle, thus the image coefficients will be relatively smaller. In between, we find the image coefficients agree quite well for $\epsilon_{1}^{V}$ for most values of $h / w$, but for $\xi_{1}^{V}$ only when $h / w \lesssim 0.7$. On the other hand, the deviation is quite large for $\xi_{1}^{H}$ for nearly all aspect ratios. This difference can be explained as follows. For horizontal oscillation of a centered beam in a rectangular vacuum chamber of smaller $h / w$, the beam can hardly be affected by its images in the far vertical end walls. On the other hand, in an elliptical vacuum chamber, because of the curved surface, it is much easier for the beam to see the changes in its image current when the beam is oscillating horizontally, so $\xi_{1}^{H}$ will be relatively larger for an elliptical cross section than a rectangular cross section at the same $h / w$. 


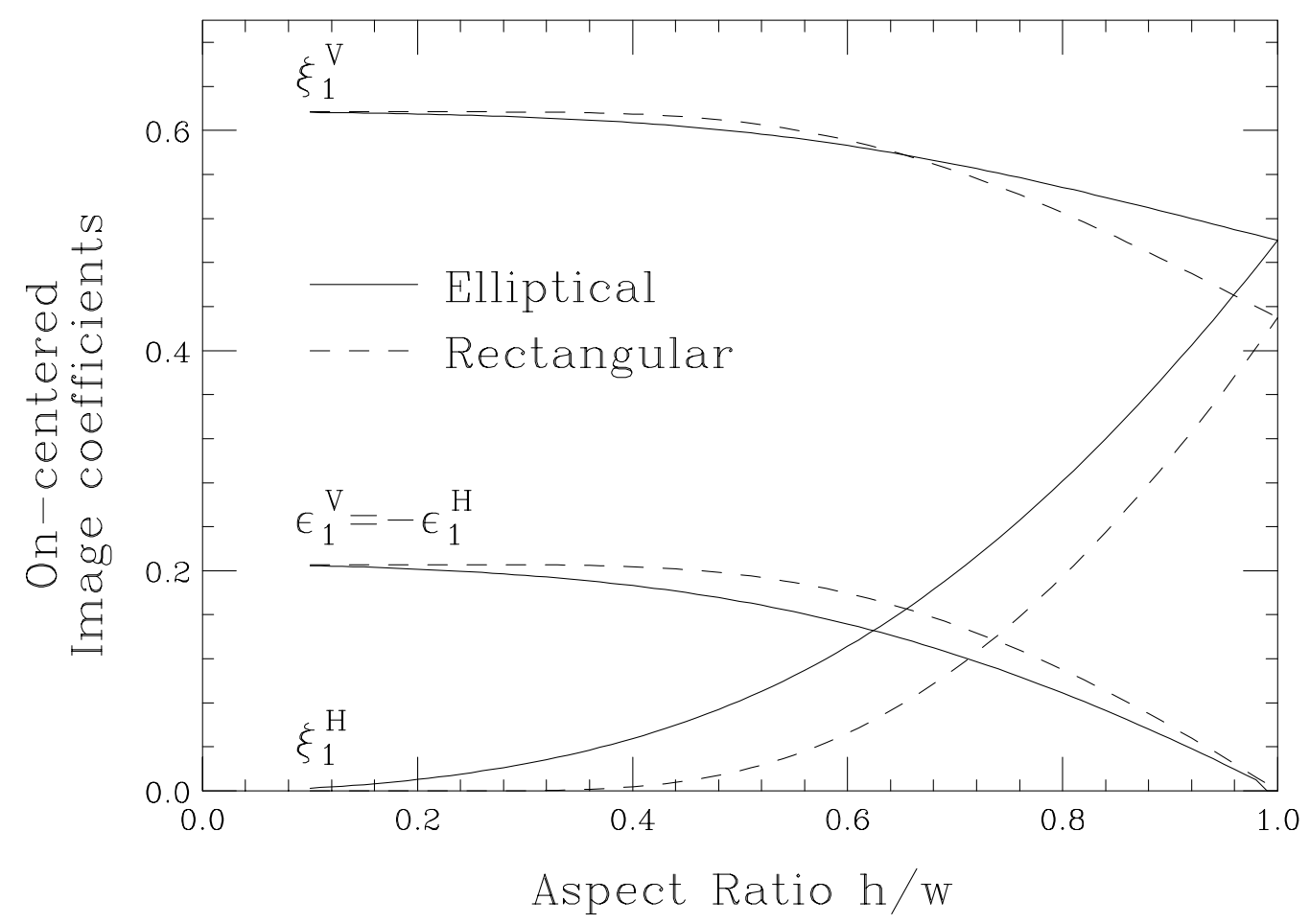

Figure 10: Comparison of the image coefficients $\epsilon_{1}^{V}, \xi_{1}^{V}$, and $\xi_{1}^{H}$ for an elliptical vacuum chamber and a rectangular chamber when the beam is on-centered. 


\section{APPENDIX}

\section{A.1 THE BEAM FORCE}

Since we are interested only in small amplitude betatron oscillations, we can Taylor expand the beam force averaged over a revolution $\left\langle F_{\text {beam }}\right\rangle$ and keep only the dipole terms. The equation of vertical motion of a test particle is therefore

$$
\frac{d^{2} y}{d s^{2}}+\frac{\left(\nu_{0}^{V}\right)^{2}}{R^{2}} y=\frac{1}{\gamma m v^{2}}\left(\left.\frac{\partial\left\langle F_{\text {beam }}\right\rangle}{\partial y}\right|_{\bar{y}=0} y+\left.\frac{\partial\left\langle F_{\text {beam }}\right\rangle}{\partial \bar{y}}\right|_{y=0} \bar{y}\right) .
$$

The average beam force depends on the vertical displacement $y$ of the test particle and also on the vertical displacement $\bar{y}$ of the center of the beam. The first term on the right side is proportional to the vertical displacement of the witness particle; it therefore constitutes a shift of the vertical betatron tune $\nu_{0}^{V}$ to become $\nu_{\text {incoh }}^{V}$. Thus, the incoherent tune shift comes from the beam force when the center of the beam is not displaced, or $\bar{y}=0$.

When Eq. (A.1) is averaged over all the particles in the beam, we get the equation of vertical motion for the center of the beam and the shift in tune will be coherent:

$$
\frac{d^{2} \bar{y}}{d s^{2}}+\frac{\left(\nu_{0}^{V}\right)^{2}}{R^{2}} \bar{y}=\frac{1}{\gamma m v^{2}}\left(\left.\frac{\partial\left\langle F_{\text {beam }}\right\rangle}{\partial y}\right|_{\bar{y}=0}+\left.\frac{\partial\left\langle F_{\text {beam }}\right\rangle}{\partial \bar{y}}\right|_{y=0}\right) \bar{y},
$$

In other words, the contribution of the coherent tune shift comes from both terms on the right side of Eq. (A.1), while the incoherent tune shift comes from only the first term.

\section{A.2 IMAGE FORMATION}

The next thing of importance is how images of the beam are formed, in the walls of the vacuum chamber? or in the magnetic pole faces? For the electric field, because the parallel component vanishes on the walls of the vacuum chamber which we assume to be infinitely conducting, images will always be formed in the walls of the vacuum chamber. We therefore say that electric field is always non-penetrating. In this discussion, penetrating or non-penetrating always implies penetrating or non-penetrating the vacuum chamber.

The magnetic field is quite different. All low-frequency magnetic field will penetrate the vacuum chamber and form images in the magnet pole faces. If no magnet pole faces 
are present, we assume that magnetic field will go to infinity and will no longer affect the test particle. All high-frequency magnetic field will not penetrate the vacuum chamber and form images in the walls of the vacuum chamber.

Before proceeding further, there is an important rule that is worth mentioning. For images in the wall of the vacuum chamber, we use the electric image coefficient $\epsilon_{1}^{V, H}$ or $\xi_{1}^{V, H}$, depending on whether it is incoherent or coherent. The electric image coefficients are used not only for electric images but also for magnetic images. The only difference is that, for magnetic images, we use $-\beta^{2} \epsilon_{1}^{V, H}$ or $-\beta^{2} \xi_{1}^{V, H}$. This is because the actual contribution of magnetic field from the images in the walls of the vacuum chamber is exactly the same as the electric field. The factor $\beta^{2}$ comes about because we need a factor of $\beta$ from the magnetic part of the Lorentz force and another factor of $\beta$ from the source which is the beam current. The negative sign comes about because the magnetic force on a beam is always in opposite direction to the electric force. As for images formed in the magnet pole faces, they can only be magnetic images, because electric field cannot penetrate the vacuum chamber. Their contributions will be $\beta^{2} \epsilon_{2}^{V, H}$ or $\beta^{2} \xi_{2}^{V, H}$, respectively, when the tune shifts are incoherent or coherent. Here we have the same factor of $\beta^{2}$. However, there is no negative sign, which is just a convention. In other words, one may consider the negative sign to have been absorbed into the definition of $\beta^{2} \epsilon_{2}^{V, H}$ or $\beta^{2} \xi_{2}^{V, H}$, We can also say that electric image coefficients are for images in the walls of the vacuum chamber independent of whether the effect is electric or magnetic, while magnetic image coefficients are for images in the magnet pole faces. All these considerations are summarized in Table II, where we also separate coherent tune shift into two parts: the dc part $\partial\left\langle F_{\text {beam }}\right\rangle /\left.\partial y\right|_{\bar{y}=0}$ when the beam is stationary and the ac part $\partial\left\langle F_{\text {beam }}\right\rangle /\left.\partial \bar{y}\right|_{y=0}$ when the beam is oscillating.

\section{A.3 TUNE SHIFTS}

\section{A.3.1 COASTING BEAMS}

Now we are ready to express the tune shifts in terms of image coefficients. First, let us study the simpler case of a coasting beam, where the only ac magnetic field comes from betatron oscillations. The frequency will be low when the betatron tune is close to

an integer and the magnetic field may be penetrating. On the other hand, the frequency will be high when the betatron tune is close to a half integer and the magnetic field may 
Table II: Relation of each component of the beam force to the image coefficients with images formed in the vacuum chamber or magnetic pole faces.

\begin{tabular}{|l|c|c|c|l|}
\hline \multirow{2}{*}{ Beam force components } & \multicolumn{2}{|c|}{$\begin{array}{c}\text { Images in } \\
\text { vacuum chamber }\end{array}$} & $\begin{array}{c}\text { Images in } \\
\text { pole faces }\end{array}$ & \multirow{2}{*}{ Comments } \\
\cline { 2 - 4 } & electric & magnetic & magnetic & \\
\hline$\left.\frac{\partial\left\langle F_{\text {beam }}\right\rangle}{\partial y}\right|_{\bar{y}=0}$ & $\frac{\epsilon_{1}^{V, H}}{h^{2}}$ & $-\beta^{2} \frac{\epsilon_{1}^{V, H}}{h^{2}}$ & $\beta^{2} \frac{\epsilon_{2}^{V, H}}{h_{m}^{2}}$ & incoherent \\
dc coherent
\end{tabular}

be non-penetrating. The incoherent tune shifts are:

$$
\Delta \nu_{\text {incoh }}^{V, H}=-\frac{N r_{0} R}{\pi \gamma \beta^{2} \nu_{0}^{V, H}}\left[\frac{\epsilon_{1}^{V, H}}{h^{2}}+\mathcal{F} \beta^{2} \frac{\epsilon_{2}^{V, H}}{h_{m}^{2}}+\left(1-\beta^{2}\right) \frac{\epsilon_{\mathrm{spch}}^{V, H}}{a_{V}^{2}}\right] .
$$

electric image magnetic image self-field, $\left(1-\beta^{2}\right)$ gives in vacuum chamber in magnet poles balance between $\vec{E}$ and $\vec{H}$

Here, the first term comes from the electric images in the vacuum chamber since electric field is always non-penetrating and therefore the incoherent electric image coefficient $\epsilon_{1}^{V, H} / h^{2}$. The second term comes the magnetic images in the magnet pole faces and therefore the incoherent magnetic image coefficient $\epsilon_{2}^{V, H}$ together with the factor $\beta^{2}$ in front and $h_{m}^{2}$ in the denominator. As stated before, the incoherent beam force comes from the images of the beam center which is not displaced or $\bar{y}=0$. These images are not moving and the beam force is therefore static or dc, and the magnetic field is therefore landing on the magnet pole faces. The last term is just the space-charge contribution, where the 1 denotes the electric part and $-\beta^{2}$ the magnetic part.

For the coherent tune shifts of a coasting beam, if the magnetic field is penetrating, 
we just have simply,

$$
\begin{array}{rr}
\Delta \nu_{\text {coh }}^{V, H}=-\frac{N r_{0} R}{\pi \gamma \beta^{2} \nu_{0}^{V, H}}\left[\frac{\xi_{1}^{V, H}}{h^{2}}+\mathcal{F} \beta^{2} \frac{\xi_{2}^{V, H}}{h_{m}^{2}}\right], \\
\uparrow & \uparrow \\
\text { electric image } & \text { magnetic image } \\
\text { in vacuum chamber } & \text { in magnet poles }
\end{array}
$$

where all the magnetic field penetrates the vacuum chamber and forms images in the magnet pole faces. Equations (A.3) and (A.4) are just Eqs. (2.1) and (2.2), respectively, when we set the bunching factor to $B=1$ and the neutralization factor to $\chi_{e}=0$. Note that there is no space-charge term because the center of the beam does not see the self-force among beam particles.

When the magnetic field is non-penetrating, we have instead

$$
\begin{aligned}
& \Delta \nu_{\mathrm{coh}}^{V, H}=-\frac{N r_{0} R}{\pi \gamma \beta^{2} \nu_{0}^{V, H}}\left[\frac{\xi_{1}^{V, H}}{h^{2}}+\mathcal{F} \beta^{2} \frac{\epsilon_{2}^{V, H}}{h_{m}^{2}}-\beta^{2} \frac{\xi_{1}^{V, H}-\epsilon_{1}^{V, H}}{h^{2}}\right] . \\
& \uparrow \quad \uparrow \quad \uparrow \\
& \text { electric image magnetic image ac magnetic image } \\
& \text { in vacuum chamber in magnet poles in vacuum chamber }
\end{aligned}
$$

To understand this expression, recall the magnetic part of beam force on the right side of Eq. (A.1). The ac field comes from the betatron oscillation of the whole beam and has its source from the second term on the right side only, since we require a moving beam center or $\bar{y} \neq 0$. According to Table II, the contribution is therefore $-\beta^{2}\left(\xi_{1}^{V, H}-\epsilon_{1}^{V, H}\right) / h^{2}$. The dc part of the coherent magnet beam force is the first term on the right side of Eq. (A.1). Since this dc field produces images in the magnet pole faces, we have therefore the second term of Eq. (A.5). The first term comes from the electric component of the coherent beam force. After re-arrangement, the coherent tune shift with penetrating fields becomes Eq. (2.3) with $B=1$ and $\chi_{e}=0$.

\section{A.3.2 BUNCHED BEAMS}

For bunched beam, we would like to compute the maximum tune shifts when the beam current is at its local maximum. We therefore divide by the bunching factor $B$ suitably so that the bunch intensity will be properly enhanced. Notice that ac magnetic field now comes from two sources: transverse betatron oscillation of the bunch and longitudinal or axial bunching of the bunch. Although both effects are ac, their 
frequencies are in general very different. The frequency of transverse betatron oscillation is $\left(n-\nu_{0}^{V, H}\right) \omega_{0} /(2 \pi)$, where $n$ is the revolution harmonic closest to the tune. These frequencies are therefore only fractions of the revolution frequency. On the other hand, the axial bunch frequency is a $h \omega_{0} /(2 \pi)$ with $h$ the rf harmonic, which is many times revolution frequency. For this reason, we consider the ac magnetic fields arising from axial bunching always non-penetrating, while the ac magnetic fields arising from betatron oscillation sometimes non-penetrating and sometimes penetrating.

In the expressions below, we try also to include the effect of trapped particles that carry charges of the opposite sign. Take a proton beam, for example, electrons can be trapped, giving a neutralization coefficient $\chi_{e}$, which is defined as the ratio of the total number of trapped electrons to the total number of protons. (For antiproton beam, the particles trapped are positively charged ions.) The trapped electrons will not travel longitudinally. Therefore, they only affect the electric force but not the magnetic force. In other words, for electric contributions, we replace 1 by $\left(1-\chi_{e}\right)$.

The incoherent tune shift for a bunched beam is expressed as

$$
\begin{aligned}
& \Delta \nu_{\text {incoh }}^{V, H}=-\frac{N r_{0} R}{\pi \gamma \beta^{2} \nu_{0}^{V, H}}\left[\frac{1-\chi_{e}}{B} \frac{\epsilon_{1}^{V, H}}{h^{2}}+\mathcal{F} \beta^{2} \frac{\epsilon_{2}^{V, H}}{h_{m}^{2}}-\beta^{2}\left(\frac{1}{B}-1\right) \frac{\epsilon_{1}^{V, H}}{h^{2}}+\left(1-\chi_{e}-\beta^{2}\right) \frac{\epsilon_{\mathrm{spch}}^{V, H}}{a_{V}^{2}}\right] . \\
& \uparrow \quad \uparrow \\
& \uparrow \quad \uparrow
\end{aligned}
$$

The second term represents magnetic fields of a stationary beam and its images and therefore the usual incoherent magnetic image coefficient $\epsilon_{2}^{V, H}$, which describes dc magnetic fields penetrating the vacuum chamber and land at the magnet poles. Here, there is no division by the bunching factor $B$, because we are talking about the dc fields coming from the average beam current.

The third term is for the ac magnetic fields generated from axial bunching and a division by $B$ is therefore necessary. Since the ac magnetic fields are non-penetrating, their contribution is the same as that of the incoherent electric field and therefore the factor $-\beta^{2} \epsilon_{1}^{V, H}$. We must remember that there is a dc part that lands on the magnet pole faces which we have considered already and must not be included here again. For this reason, we need to replace $B^{-1}$ by $B^{-1}-1$. The accuracy of this term can be inferred by noticing its disappearance when we let $B \rightarrow 1$, or the bunched beam becomes totally unbunched. After re-arrangement, this incoherent tune shift becomes Eq. (2.1). 
For coherent motion with penetrating magnetic fields from betatron oscillation, we have

$$
\begin{array}{rcc}
\Delta \nu_{\text {coh }}^{V, H}=-\frac{N r_{0} R}{\pi \gamma \beta^{2} \nu_{0}^{V, H}}\left[\frac{1-\chi_{e}}{B} \frac{\xi_{1}^{V, H}}{h^{2}}\right. & \left.+\mathcal{F} \beta^{2} \frac{\xi_{2}^{V, H}}{h_{m}^{2}}-\beta^{2}\left(\frac{1}{B}-1\right) \frac{\xi_{1}^{V, H}}{h^{2}}\right] . \\
\uparrow & \uparrow & \uparrow \\
\text { electric image } & \text { magnetic image } & \text { ac magnetic image } \\
\text { in vacuum chamber } & \text { in magnet poles } & \text { from axial bunching }
\end{array}
$$

where the third term is contributed by the magnetic field of bunching frequencies, which cannot penetrate the vacuum chamber. The magnetic fields divide into the dc part and the ac part in exactly the same way as Eq. (A.6), the expression for incoherent tune shift. Because we are talking about coherent tune shifts, the coefficients $\epsilon_{2}^{V, H}$ and $\epsilon_{1}^{V, H}$ are replaced, respectively by $\xi_{2}^{V, H}$ and $\xi_{1}^{V, H}$. After re-arrangement, the coherent tune shifts with penetrating magnetic fields from betatron oscillation becomes Eq. (2.2).

Finally, we come to ac magnetic fields that are non-penetrating coming from both axial bunching and betatron oscillation. The coherent tune shifts are

$$
\begin{aligned}
& \Delta \nu_{\text {coh }}^{V, H}=-\frac{N r_{0} R}{\pi \gamma \beta^{2} \nu_{0}^{V, H}}\left[\frac{1-\chi_{e}}{B} \frac{\xi_{1}^{V, H}}{h^{2}}+\mathcal{F} \beta^{2} \frac{\epsilon_{2}^{V, H}}{h_{m}^{2}}-\beta^{2} \frac{\xi_{1}^{V, H}-\epsilon_{1}^{V, H}}{h^{2}}-\beta^{2}\left(\frac{1}{B}-1\right) \frac{\xi_{1}^{V, H}}{h^{2}}\right] . \\
& \uparrow \uparrow \uparrow \uparrow \uparrow \\
& \text { electric image magnetic image ac magnetic image ac magnetic image } \\
& \text { in vacuum chamber in magnet poles from transverse from axial bunching } \\
& \text { motion }
\end{aligned}
$$

Here, the axial bunching parts are very exactly the same as in Eq. (A.7) because they describe exactly the same ac magnetic fields coming from axial bunching. As for the dc magnetic fields, the contribution in Eq. (A.7) come from both terms of the beam force on the right side of Eq. (A.1) and contribute the coefficient $\xi_{2}^{V, H}$. Here the dc magnetic fields come from only the first term of the beam force and contribute $\epsilon_{1}^{V, H}$ instead, for exactly the same reason as in Eq. (A.3). The part of the second term that comes from betatron oscillation of the beam gives rise to the second last term of Eq. (A.8), for exactly the same reason as in Eq. (A.3). After re-arrangement, this coherent tune shift takes the form as Eq. (2.3). 


\section{References}

[1] L.J. Laslett, Proceedings of 1963 summer Study on Storage Rings, BNL-Report 7534, p. 324; L.J. Laslett and L. Resegotti, Proceedings of VIth Int. Conf. on High Energy Accelerators, Cambridge, MA, 1967, p. 150.

[2] B. Zotter, Nucl. Instru. Meth. 129, 377 (1975).

[3] B. Zotter, CERN Report ISR-TH/74-11 (1974).

[4] B. Zotter, CERN Reports ISR-TH/72-8 (1972), IST-TH/74-38 (1974), ISR-TH/7517 (1975); B. Zotter, Proceedings of VIth National particle Accelerator Conf., Washington DC, 1974 (IEEE, 1975).

[5] See, for example, Table 16.5, p.571 of Abramowitz and Stegun, Handbook of Mathematical Functions, Dover, 1965.

[6] K.Y. Ng, Particle Accelerators 16, 63 (1984). 